\title{
Energy Efficiency about Collaborative MIMO Technology
}

\author{
Huajun Chen ${ }^{1, a}$, Lina Yuan ${ }^{1, b}$ \\ ${ }^{1}$ School of Information Engineering, Tongren University, Guizhou ,554300, China

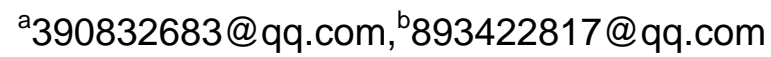

Keywords: wireless sensor nodes; energy consumption; throughput; Multi-input multi-output (MIMO) systems; single-input single-output (SISO) systems; wireless sensor networks (WSNs)

\begin{abstract}
Main energy consumption of wireless sensor nodes is used to send and receive data. To take measures to reduce sending and receiving energy consumption of nodes plays an important role in decreasing energy consumption of the whole network. Researches show that: under the requirements of the same throughput, sending the same information, dissipative energy of Multiinput multi-output (MIMO) systems are less than single-input single-output (SISO) systems. Therefore, introducing the MIMO technology in the wireless sensor networks (WSNs) can save the sending energy consumption of the network.
\end{abstract}

\section{Introduction}

Multi-input multi-output (MIMO) technology adopts multiple antennas and multi-channel technology in the transmitting and receiving terminal of communication system through multiple antennas to send and receive data together. Research results show that multi-input multi-output (MIMO) technology can exponentially increase the channel capacity in the communication system without any increase in channel spectrum resources and antenna with sending power. Based on this, MIMO technology is also widely used for a new generation of mobile communication system.

\section{Research on energy consumption of MIMO systems}

MIMO systems can support higher data transmission rate than SISO systems ${ }^{[1]}$ in the performance requirement of the same transmission power and the same bit error rate. For the same throughput-requirement, MIMO systems needed transmitted energy are smaller than SISO systems. The literature [2]-[6] have proposed some energy optimization technique based on SISO system, studied the multimodal operating on systems with parameters optimization. The problem in the use of MIMO systems is more important, because the circuit structures of MIMO systems are more complicated. In wireless sensor network (WSN), compared with transmitted energy loss, the circuits-energy loss of the network itself can't be ignored, and due to the circuit structures of MIMO systems are more complicated, the circuits-energy loss is also higher. Therefore, although the use of MIMO technology can save more transmitted energy, because of the increase of circuit complexity, energy consumption of the circuits will also increase, which can't be sure whether the energy efficiency of MIMO systems is higher than SISO systems. This article will give the performance of MIMO systems compared with the traditional SISO systems.

\subsection{Study power loss of power amplifier}

The power loss of each sensor nodes is mainly composed of two parts: power loss of power amplifier

$p_{p a}$ and power loss of other circuits-modules $p_{c}$.

Firstly, calculate power loss of power amplifier $p_{p a}, p_{p a}$ is a function ${ }^{[7]}$ about transmitted power $p_{\text {out }}$ :

$$
p_{p a}=(1+\alpha) p_{\text {out }}
$$


Among $\alpha=\varepsilon / \eta-1, \varepsilon$ is peak-to-average ratio (PAR), and $\eta$ is drain efficiency of radio frequency power amplifier. Assuming that models work in the square-law power path attenuation of Rayleigh fading channel, through the relationship of link budget the value of $p_{\text {out }}$ can be got ${ }^{[8]}$ :

$$
p_{\text {out }}=\frac{\overline{E_{b}} R_{b}(4 \pi d)^{2}}{G_{T} G_{R} \lambda^{2}} M_{l} N_{f}
$$

In the formulation of (2), $R_{b}$ is bit rate of transmission; $d$ is the distance of transmission; $G_{T}, G_{R}$ are separately the antenna gain of transmitter and receiver; $\lambda$ is the wave length of carrier; $M_{l}$ is process changes of hardware and other additive background noise or other interferential connection edge compensation; $N_{f}$ is noise index of receiver, $N_{f}=N_{r} / N_{0} ; N_{r}$ is effective input noise power spectral density of receiver; $\overline{E_{b}}$ is the receiver receiving needed energy every bit of information under the requirement of a given bit error rate.

Assuming that models work in the square-law power path attenuation of Rayleigh fading channel, the attenuation factor of MIMO channel can be represented with a scalar matrix. Path loss can be used with a power drop model with ratio of the square of the transmission distance. Instantaneous received $\mathrm{SNR}^{[9]}$ is

$$
\gamma_{b}=\frac{\|H\|_{F}^{2}}{M_{t}} \frac{\overline{E_{b}}}{N_{0}}
$$

Among $\mathrm{H}$ is a channel matrix, each factor is zero mean cyclic symmetry complex Gaussian random variable; $N_{0}$ is the unilateral thermal noise power spectrum density at room temperature; Generally speaking, $N_{0}=-171 \mathrm{dBm} / \mathrm{Hz}, M_{t}$ is the number of collaboration nodes at the transmitting terminal.According to the literature [9], it can get the average bit error rate:

$$
\overline{P_{b}}=\varepsilon_{H}\left\{Q\left(\sqrt{2 \gamma_{b}}\right)\right\}
$$

And in line with Chernoff bound ${ }^{[9]}$ :

$$
\overline{P_{b}} \leq\left(\frac{\overline{E_{b}}}{M_{t} N_{0}}\right)^{-M_{t}}
$$

On the basis of the above formulas, it can acquire:

$$
\overline{E_{b}} \leq\left(\frac{M_{t} N_{0}}{\bar{P}_{b}^{1 / M_{t}}}\right)^{-M_{t}}
$$

Let the above boundary value approximately equal, in accordance with the formula (2), it can obtain the transmitted power $p_{\text {out }}$ :

$$
P_{\text {out }}=\frac{R_{b} N_{0} M_{t}(4 \pi d)^{2}}{P_{b}^{1 / M_{t}} G_{T} G_{R} \lambda^{2}} M_{l} N_{f}
$$

Therefore, the power consumption of power amplifier $P_{p a}$ is:

$$
P_{p a}=(1+\alpha) \frac{R_{b} N_{0} M_{t}(4 \pi d)^{2}}{P_{b}^{1 / M_{t}} G_{T} G_{R} \lambda^{2}} M_{l} N_{f}
$$

\subsection{Study power loss of other circuits-models} circuit

Figure 1 and figure 2 are separately the structural drawing of a transmitter and a receiver with

module about each sensor node. 


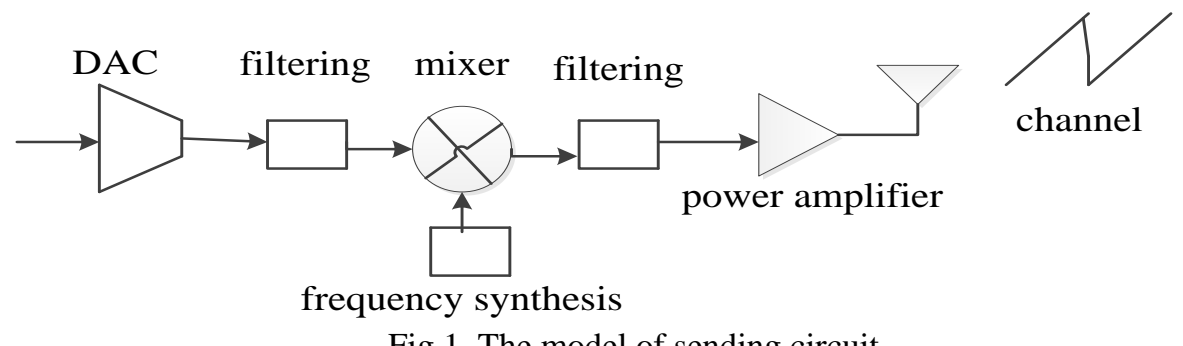

Fig.1. The model of sending circuit

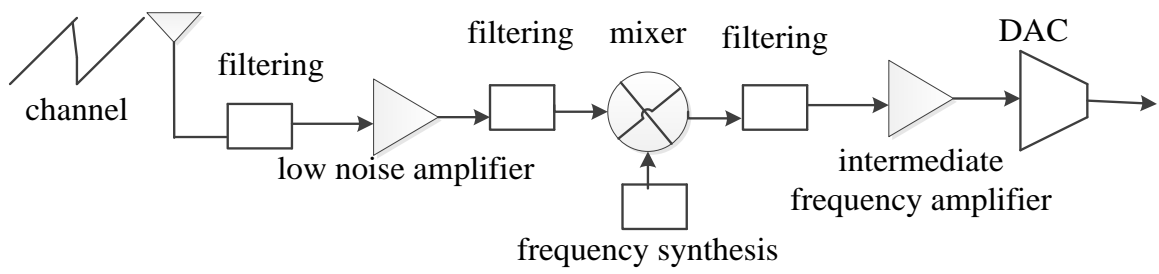

Fig.2. The model of receiving circuit

From the above two figures to be seen, energy consumption of circuits-models is:

$$
P_{C}=M_{t}\left(p_{D A C}+P_{\text {mix }}+P_{\text {filt }}\right)+2 P_{\text {syn }}+M_{r}\left(P_{A D C}+P_{\text {mix }}+P_{\text {filr }}+P_{L N A}+P_{I F A}\right)
$$

In the formula (9), $p_{D A C}, P_{\text {mix }}, P_{\text {filt }}, P_{\text {syn }}, P_{A D C}, P_{\text {filr }}, P_{L N A}, P_{I F A}$ are separately digital and analog converter, mixer, the active filter at the transmitting end, frequency synthesizer, analog and digital converter, the active filter at the receiving end, the low noise amplifier, the processing power of intermediate frequency amplifier; $M_{t}$ and $M_{r}$ are respectively the number of collaborative nodes at the transmitting and receiving end.

Finally, considering overall energy loss of the cooperative transmission system, energy value is equal to the power by time. When only send 1 bit of data, the total energy consumption $E_{b t}$ is equivalent to

$$
E_{b t}=\left(P_{p a}+P_{c}\right) T_{s}=\frac{\left(P_{p a}+P_{c}\right)}{R_{b}}
$$

Among $T$ is the needed time of sending 1 bit of data. Depending on the form (10) and the deduced formula, it can be available to the total energy consumption of sending 1 bit of information in the MIMO systems- $E_{b t}$ :

$$
E_{b t}=(1+\alpha) \frac{M_{t} N_{0}}{\bar{P}_{b}^{1 / M_{t}}} \times \frac{(4 \pi d)^{2}}{G_{T} G_{R} \lambda^{2}} M_{l} N_{f}+\frac{P_{C}}{R_{b}}
$$

\section{Compare the performance of MIMO systems with SISO systems}

First of all, from the perspective of transmission energy consumption to compare the performance of MIMO systems with SISO systems, and then from the view of the total energy to compare with them, it tries hard to win a comprehensive understanding of the performance of MIMO systems can get advantage.

MIMO systems use diversity coding of Alamouti and the modulation mode of BPSK. For MIMO, sending nodes of $M_{t}$ first broadcast their information to other local nodes in different time slot. After each node receives all bits of information from other nodes, they are coding according to the transmission sequence based on Alamouti diversity code.

Because each node has a established tag in advance-I, they will send information what the antenna of I should launch according to the transmission sequence. At the receiving end, receiving nodes of $M_{r}$ (one destination node and subsidiary nodes of $M_{r}-1$ ) participate in the collaborative receiving. Subsidiary nodes of $M_{r}-1$ first quantize each symbol what they have received into bits 
of $n_{r}$, and then use the uuencoded MQAM to put all the bits to send to the destination nodes for joint detection.

\subsection{The comparison of transmitted energy loss}

First, from the perspective of transmission energy consumption to compare the performance of MIMO systems with SISO systems, from the above discussion to be known, MIMO systems need transmitting energy of sending 1 bit of information:

$$
E_{\text {tran }}=(1+\alpha) \frac{M_{t} N_{0}}{\bar{P}_{b}^{1 / M_{t}}} \times \frac{(4 \pi d)^{2}}{G_{T} G_{R} \lambda^{2}} M_{l} N_{f}
$$

If calculating the transmitting energy consumption of SISO systems, only order the above formula- $M_{t}=1$.

This paper uses the parameters of the literature [10], specific parameters are shown in table 1.

Table 1 The parameters of systems

\begin{tabular}{|c|c|c|c|}
\hline$f_{c}=2.5 \mathrm{GHz}$ & $\eta=0.35$ & $G_{T} G_{R}=5 \mathrm{dBi}$ & $\sigma^{2}=\frac{N_{0}}{2}=-174 \mathrm{dBm} / \mathrm{Hz}$ \\
\hline$B=30.3 \mathrm{KHz}$ & $\varepsilon=0.5$ & $p_{\text {mix }}=30.3 \mathrm{~mW}$ & $p_{\text {syn }}=50 \mathrm{~mW}$ \\
\hline$p_{b}=10^{-3}$ & $T_{s}=\frac{1}{B}$ & $P_{\text {filt }}=P_{\text {fil }}=2.5 \mathrm{~mW}$ & $P_{L N A}=20 \mathrm{~mW}$ \\
\hline$P_{D A C}=15.5 \mathrm{~mW}$ & $P_{I F A}=3 \mathrm{~mW}$ & $N_{f}=10 \mathrm{~dB}$ & $M_{l}=40 \mathrm{~dB}$ \\
\hline
\end{tabular}

Another assumption that $R_{b}=10 \mathrm{kbit} / \mathrm{s}$, we first compare the transmission energy consumption of MIMO systems with SISO systems by changing the transmission distanced under the condition of different transmission distance. In addition, supposing $M_{l}=2$ in MIMO systems, namely, there are only two transmitting antenna at the sending end.

As can be seen from the figure 3, when just compare the transmission energy consumption of MIMO systems with SISO systems, the former have higher energy efficiency. And with the increase of transmission distance, MIMO systems compared can save more and more energy than SISO systems. Figure 3 can fully show the superiority in the aspect of improving the efficiency of energy transmission in MIMO systems.

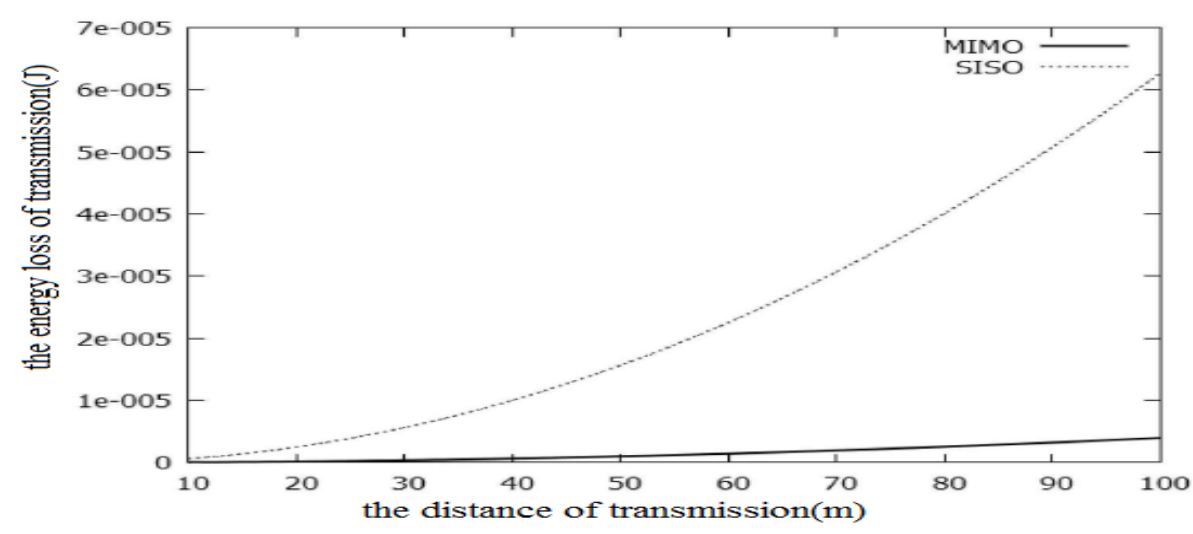

Fig. 3.

\subsection{The comparison of total energy loss}

From the above, it can be known that MIMO systems have significant effect on improving the efficiency of transmission energy. In the long distance of transmission, the transmission energy consumption dominates in the total energy consumption. In the applications of short distance, however, such as in sensor network, energy consumption of the circuits such as hardware in the network is not less than transmission energy consumption, and even it may be dominant. So in WSN, compared with transmission energy loss, the network itself can't be ignored, and due to the 
circuit structures of MIMO systems are more complex, the use of energy loss of circuits in MIMO systems is also higher. Therefore, although using MIMO technology can save more energy, due to the increase in energy consumption of circuits, it cannot be sure whether energy efficiency of MIMO systems is higher than SISO systems. This section will give the performance of MIMO systems compared with the traditional SISO systems.

From the above research, it can be known that MIMO systems need energy consumption of sending 1 bit of information- $E_{b t}$ :

$$
E_{b t}=(1+\alpha) \frac{M_{t} N_{0}}{\bar{P}_{b}^{1 / M_{t}}} \times \frac{(4 \pi d)^{2}}{G_{T} G_{R} \lambda^{2}} M_{l} N_{f}+\frac{P_{C}}{R_{b}}
$$

In this section, still use the parameters in table 1 , and assume $R_{b}=10 \mathrm{kbit} / \mathrm{s}$.Provided that in MIMO systems, $M_{t}=2$ and $M_{r}=2$, that is to say, only two antenna participate in collaboration at the transmitting and receiving end.

Figure 4 shows that for single jump network, when the transmission distance is greater than a certain threshold, energy efficiency of cooperative MIMO systems is higher than SISO systems. This is because, when the transmission distance is larger, energy consumption of transmission also increases in proportion in total energy consumption accordingly. While the circuit structures of cooperative MIMO systems are more complex, it brings the additional energy consumption of circuits, but when the transmission distance reaches a certain threshold, transmission energy of cooperative MIMO will be greater than the additional energy consumption of circuits for complex. Therefore, In this case the cooperative MIMO saves more energy efficient than SISO. In this example, the threshold value of $\mathrm{d}$ equals $48 \mathrm{~m}$. After the transmission distance is greater than the threshold, with the increasing of transmission distance, energy-saving advantages of MIMO systems became more and more compared with the SISO systems. For the requirements of the same throughput, the transmission energy what MIMO systems need is less than SISO systems.

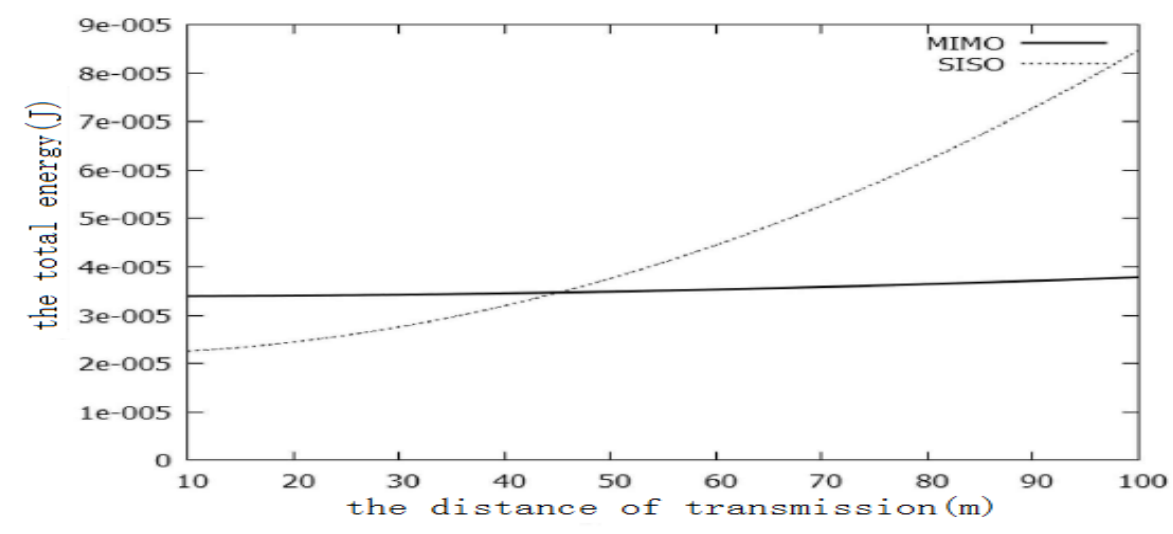

Fig. 4.

\section{Summary}

This paper first analyses the energy consumption models of transmission and Circuits in MIMO Systems, and then compares the energy consumption models of transmission in MIMO systems with that of SISO systems, whose results find that the energy transmission efficiency in MIMO systems is better than that of SISO systems. But with energy loss of circuits, due to the circuit structures of MIMO systems are more complex, so when the transmission distance is shorter, energy loss of circuits in MIMO systems is higher, and accounts for more and more proportion in the total energy, the total energy consumption is higher than that of SISO systems. But when the transmission distance increases, the energy loss of transmission accounts for more and more proportion in the total energy, MIMO systems reflect the advantages in the field of saving transmission energy. When the saving transmission energy in MIMO systems is greater than the 
additional energy consumption of circuits, MIMO systems become more efficient. So there exists a threshold, when the transmission distance is greater than the threshold, energy efficiency of MIMO systems is higher than that of SISO systems.

\section{Acknowledgment}

This work is supported by the Collaborative Fund Project of Science and Technology Agency in Guizhou Province marked by the word LH on 7487 [2014] and the reform project of teaching contents and curriculum system in colleges and universities of Guizhou Province on 2014 SJJGXM003.

\section{References}

[1]A.Paulraj,R.Nabar,D.Gore,Introduction to Space-Time Wireless Communications[M], Cambridge, U.K.:Cambridge Univ. Press, 2003.preprint.

[2]C.Schurgers, O. Aberthorne, M. B. Srivastava. Modulation scaling for energy aware communication systems,[C]. in Proc. Int. Symp. Low Power Electronics Design, Aug. 2001, p.9699.

[3]R Min,A.Chandrakasan. A framework for energy-scalable communication in high-density wireless networks,[C]. in Proc. Int. Symp. Low Power Electronics Design, Aug.2002,p.36-41.

[4]S.Cui,A.J.Goldsmith, A. Bahai. Modulation optimization under energy constraints,[J]. in Proc.ICC'03,AK,May2003,p.2805-

2811.[Online].Available:http://wsl.stanford.edu/Publications.html.

[5]S.Cui,A.J.Goldsmith, A. Bahai. Energy-constrainedmodulation optimization for coded systems[J].in Proc.GLOBECOM'03,SanFrancisco,CA,Dec.2003,p.372-376.[Online].Available: http://wsl. stanford. edu/ Publications.html.

[6]S.Cui,A.J.Goldsmith,A.Bahai.Energy-constrained modulation optimization[J].IEEE Trans. Wireless Commun., 2004. [Online]. Available: http://wsl.stanford.edu/Publications.html, submitted for publication.

[7]S.Cui,A.J.Goldsmith,A.Bahai.Energy-constrained modulation optimization,[C].IEEE Trans. Wireless Commun.,vol.4,no.9(2005),p.2349-2360.

[8] J. G. Proakis. Digital Communications [M], 4th ed. New York: McGraw-Hill, 2000.

[9]A.Paulraj,R. Nabar, D.Gore.Introduction to Space-Time Wireless Communications[M],Cambridge, U.K.:Cambridge Univ.Press,2003.preprint.

[10]Cui Shuguang,A J Goldsmith,A Bahai.Energy efficiency of MIMO and cooperative MIMO techniques in sensor networks[J].IEEE Journal on selected Areas in communications,vol 22(2004)p.1089-1098.

[11]ZHANG GuoPeng,Pareto optimal time-frequency resource allocation for selfish wireless cooperative multicast networks[J]. Science China(Information Sciences),Vol.56(2013)122306:1122306:8.

[12]Gregori, M. and M. Payaro, Energy-Efficient Transmission for Wireless Energy Harvesting Nodes. IEEE Transactions on Wireless Communications[J],vol.12(2013): p.1244-1254.

[13]H.Zhang,A.Gladisch,M.Pickavet,Z.Tao,and W. Mohr,Energy efficiency in communications, IEEE Commun. Mag.[J],vol. 48(2010),p.48-49 .

[14]Lina Yuan, Jing Gong,An Analysis of Large-scale Wireless Sensor Networks with Energysaving Problem[J].Applied Mechanics and Materials, Vol 686 (2014) p.412-416. 